Draft version August 6, 2015

Preprint typeset using LTEX style emulateapj v. 5/2/11

\title{
THE SOLAR SYSTEM AS AN EXOPLANETARY SYSTEM
}

\author{
Rebecca G. Martin ${ }^{1}$ and Mario Livio ${ }^{2}$ \\ ${ }^{1}$ Department of Physics and Astronomy, University of Nevada, Las Vegas, 4505 South Maryland Parkway, Las Vegas, NV 89154, USA and \\ ${ }^{2}$ Space Telescope Science Institute, 3700 San Martin Drive, Baltimore, MD 21218, USA \\ Draft version August 6, 2015
}

\begin{abstract}
With the availability of considerably more data, we revisit the question of how special our Solar System is, compared to observed exoplanetary systems. To this goal, we employ a mathematical transformation that allows for a meaningful, statistical comparison. We find that the masses and densities of the giant planets in our Solar System are very typical, as is the age of the Solar System. While the orbital location of Jupiter is somewhat of an outlier, this is most likely due to strong selection effects towards short-period planets. The eccentricities of the planets in our Solar System are relatively small compared to those in observed exosolar systems, but still consistent with the expectations for an 8-planet system (and could, in addition, reflect a selection bias towards high-eccentricity planets). The two characteristics of the Solar System that we find to be most special are the lack of super-Earths with orbital periods of days to months and the general lack of planets inside of the orbital radius of Mercury. Overall, we conclude that in terms of its broad characteristics our Solar System is not expected to be extremely rare, allowing for a level of optimism in the search for extrasolar life.

Subject headings: planetary systems - planets and satellites: formation - protoplanetary disks
\end{abstract}

\section{INTRODUCTION}

The discovery of thousands of extrasolar planets and planet candidates in recent years (see, e.g., Wright et al. 2011; Batalha 2014; Rowe et al. 2014; Lissauer, Dawson \& Tremaine 2014; Han et al. 2014, and references therein and see exoplanet.org for a complete list), coupled with the rapidly increasing interest in the potential existence of extrasolar life, raise again in a big way the question of whether or not our Solar System is special in any sense. More specifically, we are interested in understanding whether the planetary and orbital properties in our Solar System are typical or extremely unusual compared to those of extrasolar planets.

The Solar System contains eight planets and two main belts (the asteroid belt and the Kuiper belt). While tens of debris disks and warm dust belts (similar perhaps to the Solar System's asteroid belt) have been observed and resolved, belts with dust masses as low as those in the Solar System would currently be undetectable in extrasolar systems (e.g. Wyatt 2008; Panić et al. 2013). Consequently, we can quantitatively assess in detail how special the Solar System is, only on the basis of its planetary components and properties such as its age and metallicity. However, there are now hundreds of unresolved debris disk candidates (e.g. Chen et al. 2014). Of these, about two thirds of the systems are better modelled by a two component dust disk rather than a single dust disk. The two temperature components likely arise from two separate belts (Kennedy \& Wyatt 2014). Thus, the two belt configuration of our own Solar System is plausibly fairly typical.

Beer et al. (2004) made an initial attempt to explore to what extent Jupiter's periastron could be considered atypical compared to those of the giant planets known at the time. Their analysis, however, included only fewer than 100 exoplanets, most of which had been detected via radial velocity measurements. Consequently, selection effects dominated their conclusions - a possibility fully acknowledged by the authors. In the present work we re-examine the question of how special the Solar System is. In Section 2 we use the much larger currently available database to consider the planetary orbital parameters. We identify the semi-major axis of the innermost planet as the most discrepant characteristic of the solar system and the low mean eccentricity as being somewhat special. In Section 3 we compare the masses and densities of the planets in our Solar System with those in exosolar systems. While the lack of a super-Earth in the Solar System is somewhat unusual, we argue that none of the characteristics identified make the Solar System very special. We discuss potential implications of our results in Section 4. Some of the apparent differences between the Solar System and exoplanetary systems continue to be driven by strong selection effects that affect the sample. We draw our conclusions in Section 5.

\section{PLANETARY ORBITAL PROPERTIES}

We first consider the statistical distribution of orbital separation and eccentricity of the observed planetary orbits. To allow for a more meaningful quantitative analysis, we perform a Box \& Cox (1964) transformation on the data. This transformation makes the data closer to a normal distribution so that we can more accurately evaluate properties such as the mean and the standard deviation. The transformation takes a skewed data set to approximate normality. It is based on the geometric mean of the measurements and is independent of measurement units. It is possible to do a multivariate BoxCox transformation (e.g. Velilla 1993). However, because of the selection effects associated with different parameters we choose to consider each parameter separately.

We transform the data with the function

$$
y_{\lambda}(a)= \begin{cases}\frac{a^{\lambda}-1}{\lambda} & \text { if } \lambda \neq 0 \\ \log a & \text { if } \lambda=0\end{cases}
$$

where $a$ is the parameter we are examining, such as the semimajor axis or eccentricity, and $\lambda$ is a constant that depends upon the original distribution, that we discuss below. The maximum likelihood estimator of the mean of the transformed data is

$$
\bar{y}_{\lambda}=\sum_{i=1}^{n} \frac{y_{\lambda, i}}{n},
$$


where $y_{\lambda, i}=y_{\lambda}\left(a_{i}\right)$ and $a_{i}$ is the $i$-th measurement of a total of $n$. Similarly, the maximum likelihood estimator of the variance of the transformed data is

$$
s_{\lambda}^{2}=\sum_{i=1}^{n} \frac{\left(y_{\lambda, i}-\bar{y}_{\lambda}\right)^{2}}{n}
$$

We choose $\lambda$ such that we maximise the log likelihood function

$$
l(\lambda)=-\frac{n}{2} \log (2 \pi)-\frac{n}{2}-\frac{n}{2} \log s_{\lambda}^{2}+(\lambda-1) \sum_{i=1}^{n} \log a_{i} .
$$

This new distribution, $y_{\lambda}(a)$, will be an exact normal distribution if $\lambda=0$ or $1 / \lambda$ is an even integer.

We can measure how well the transformed distribution compares to a normal distribution with two parameters. The skewness is

$$
S=\sum_{i=1}^{n} \frac{1}{n}\left(\frac{y_{\lambda, i}-\bar{y}_{\lambda}}{\sigma}\right)^{3},
$$

where $\sigma$ is the standard deviation of the distribution and thus we take $\sigma=s_{\lambda}$. The skewness measures the asymmetry of the distribution, a positive number implying the right hand tail of the distribution is longer, and a negative number that the left hand tail is longer. Furthermore, we can compare the kurtosis,

$$
K=\sum_{i=1}^{n} \frac{1}{n}\left(\frac{y_{\lambda, i}-\bar{y}_{\lambda}}{\sigma}\right)^{4}-3
$$

This is a measure of the "peakedness" of the distribution and the heaviness of the tails. A normal distribution has a kurtosis value of zero. A positive value means that the distribution is tightly peaked but the tails are broad, and vice versa for a negative value. In the following subsections we take the samples of the eccentricity and semi-major axis of the observed exoplanets and compare them to the planets in our own Solar System.

\subsection{Eccentricity}

There are a total of 539 exoplanets with a measured eccentricity. In the left hand panel of Fig. 1 we show the eccentricity distribution for this sample. We find the maximum of the $\log$ likelihood function to be when $\lambda=0.30$. The right hand panel of Fig. 1 shows the histogram of the BoxCox transformed data. For our data, we find the skewness to be $S=-0.065$. For a normal distribution we expect the magnitude of the skewness to be up to $\sqrt{6 / n}=0.11$. Thus the data are not heavily skewed. The kurtosis is $K=-0.53$ whereas for a normal distribution we would expect the magnitude to have values up to $\sqrt{24 / n}=0.21$. Thus, the data have a slightly large kurtosis, or the distribution is not so tightly peaked as a normal one. For the transformed data, the mean is -1.42 and the standard deviation is 0.60 . Jupiter lies at $-0.97 \sigma$ from the mean. Similarly the Earth lies at $-1.60 \sigma$. Thus, the eccentricities of the planets in our Solar System (that range from Venus at $e=0.0068$ to Mercury at $e=0.21$ ) are all relatively small compared to those of exoplanets, but not altogether significantly different.

Recently Limbach \& Turner (2014) considered how the mean eccentricity of planets in a system is correlated with the number of planets in the system. They found a strong anti-correlation of eccentricity with multiplicity in systems observed by radial velocity. An extrapolation of their relation up to 8 planets fits well with the mean eccentricity observed in the Solar System. Furthermore, Van Eylen \& Albrecht (2015) used the Kepler exoplanets with asteroseismically determined stellar mean densities to derive a rather low eccentricity distribution of the multi-planet Kepler systems. Thus, while the eccentricities in our Solar System are low, those may be expected in a system with so many planets.

There is some bias in the exoplanet eccentricity data. For the RV planets, the best fit eccentricity is biased upwards from the true value leading to a reduced number of systems with a low eccentricity (e.g. Shen \& Turner 2008; Hogg, Myers \& Bovy 2010; Zakamska, Pan \& Ford 2011; Moorhead et al. 2011). However, the detection efficiency decreases only mildly with increasing eccentricity because despite being more difficult to detect, they have a larger RV amplitude for a fixed planet mass and semi-major axis (Shen \& Turner 2008). While planets found with the transit method require follow up observations (for example, with $\mathrm{RV})$ to determine the eccentricity, the distribution of eccentricities is consistent with those of the RV planets (Kane et al. 2012). Without the bias, the eccentricities of the planets in our Solar System would appear to be less special.

\subsection{Semi-Major Axis}

To date, there are a total of 1580 planets with a determined semi-major axis. This increases up to a total of 5289 if we include planet candidates from Kepler. The false positive rate of the Kepler exoplanets is low, especially for multi planet systems and the non-giant planets (e.g. Désert et al. 2015) and thus we consider this much larger dataset also. We find the maximum of the log likelihood function to be when $\lambda=-0.29$ ( $\lambda=-0.20$, including planet candidates). Fig. 2 shows the histogram of the Box-Cox transformed data. The left hand panel includes only confirmed exoplanets. For this data, we find the skewness to be $S=0.11$. This is only slightly higher than the upper value expected for a normal distribution of $\sqrt{6 / n}=0.062$. We find the kurtosis to be $K=-0.48$ whereas for a normal distribution we would expect magnitudes smaller than $\sqrt{24 / n}=0.12$. Thus, the data have a large kurtosis. For the transformed data, the mean is -2.77 and the standard deviation is 2.16. Thus, Jupiter lies at $1.9 \sigma$. The right hand panel of Fig.2 repeats this analysis but includes unconfirmed Kepler planets. The skewness for this is small at $S=0.022$ (expected magnitude less than 0.034) and the kurtosis is much smaller also, $K=-0.18$ (expected magnitude less than 0.067). Jupiter lies at $2.4 \sigma$, suggesting on the face of it that Jupiter is rather special. However, as we explain below, this is most likely a result of selection effects.

The majority of the planets in this distribution have been found by transit methods. The planet with the largest semi-major axis found by this method is only at $0.996 \mathrm{AU}$ (the planet is KIC 11442793 h, Cabrera et al. 2014). Kepler, is thought to be complete only for planets at least as large as the Earth and for orbital periods up to a year (e.g Winn \& Fabrycky 2014). Microlensing surveys preferentially find planets at radial distances of a few AU from their host star, that is often an M dwarf (e.g Gould et al. 2010; Cassan et al. 2012). This scale is dictated by the size of the Einstein ring radius around the lensing star. The radial velocity method has found planets in the range 0.01 to 5.83 AU (e.g. Bonfils et al. 2013). Direct imaging can detect planets at much larger distances (e.g. Marois et al. 2008; Lafrenière, Jayawardhana \& van Kerkwijk 2010), but so far 

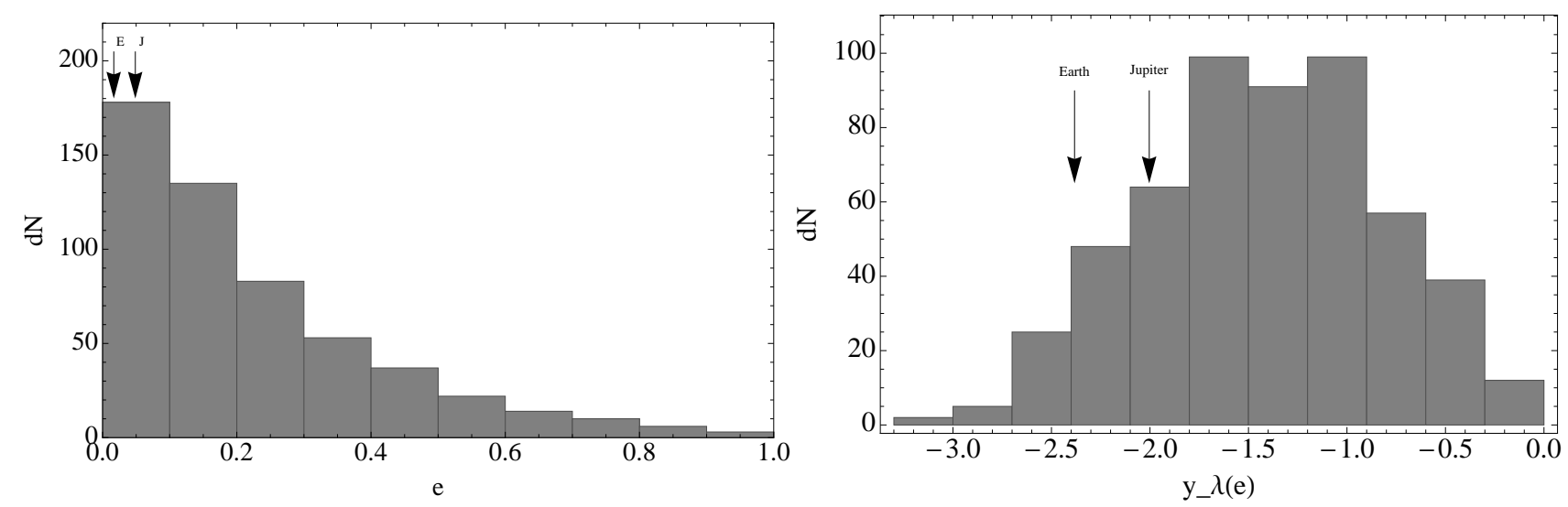

FIG. 1.- Left: Eccentricity distribution for all observed exoplanets with a measured orbital eccentricity. Right: Box-Cox transformed distribution of exoplanet eccentricities. The total number of exoplanets is 539 .

only 8 planets have been detected by the method.

In order to test the possibility that Jupiter's outlier status is largely due to selection effects, we repeated the analysis but removed planets found by the transit method. There remain 473 planets in the sample. We find the maximum of the log likelihood function to be when $\lambda=0.11$. Fig. 3 shows the histogram of the transformed data. For our data, we find the skewness to be $S=0.015$. This is less than the value expected for a normal distribution of $\sqrt{6 / n}=0.11$. For our data we find a high kurtosis of $K=0.71$ whereas for a normal distribution we would expect values with magnitude less than $\sqrt{24 / n}=$ 0.23 . For the transformed data, the mean is -0.22 and the standard deviation is 1.41 . Thus, Jupiter lies at $1.44 \sigma$ and continues to be somewhat of an outlier, but the trend suggests that this is most likely still due to selection effects. The fact that direct imaging repeatedly reveals planets at separations much larger than Jupiter's also may indicate that the current relative dearth of planets at large separations could be due to selection biases but more complete observations are required to test this possibility.

We should also note that Beer et al. (2004) used only the planet with the largest velocity semi-amplitude in each observed system in their plots. However, they also performed the analysis with the most massive planet in each system and again with all the planets. They reported no difference in the significance of Jupiter as an outlier. In 2004, they found that Jupiter was at $2.3 \sigma$ and half a sigma from its nearest neighbour. We find that Jupiter is not such an outlier as it was with the much smaller data set in 2004, and selection effects continue to affect the distribution. This analysis should again be repeated once we have more reliable observations around the orbital radius of Jupiter.

\subsection{Inner Solar System}

Currently, our inner Solar System appears to be rather special compared to observations of exoplanet systems. The inner edge of our Solar System is at the orbit of Mercury at $0.39 \mathrm{AU}$, while exoplanetary systems are observed to habor planets much closer to their star. We find that Mercury lies at $0.78 \sigma$ above the mean, while the Earth is at $1.28 \sigma$ above the mean of the distribution of confirmed exoplanet semi-major axes (as shown in the left hand panel of Fig. 2). When we include all of the Kepler candidates (right hand panel of Fig. 2), these increase to $0.98 \sigma$ for Mercury and $1.57 \sigma$ for the Earth. Thus, all of the planets in our Solar System have orbital semimajor axes that are larger than the mean observed in exoplan- etary systems. However, it is possible that this could be the result of selection effects as it is easier to find planets in this region, if they are there. In terms of the radial location of observed exoplanets, the lack of close-in planets in our Solar System is the parameter that makes our Solar System most special. We should note though that if we use only the planets found by methods other than the transit, then Mercury is at $-0.48 \sigma$ and the Earth is at $0.16 \sigma$. Consequently it is difficult to say how significant this discrepancy is.

Batygin \& Laughlin (2015) suggested that the migration of Jupiter and Saturn into the terrestrial planet forming region of our Solar System (down to $a \approx 1.5 \mathrm{AU}$ ) led to the depletion of mass in $a<0.39 \mathrm{AU}$. The planets are then thought to migrate outwards to their current location (see also Walsh et al. 2011). However, whatever the formation mechanism for the giant planets in our Solar System might have been, it is not thought to have been specific to our Solar System, and thus neither is a depleted inner Solar System. We discuss this point further in Section 4

\subsection{Migration}

Finally in this Section, we note that migration of planets through the protoplanetary disk or planet-planet interactions or secular interactions of a binary star could affect these distributions, especially that of the semi-major axes. For example, it may be theoretically impossible for Jupiter mass planets to form at the radial location of hotJupiters (e.g. Bodenheimer, Hubickyj \& Lissauer 2000). Instead, they are supposed to form outside of the snow line and migrate inwards through the protoplanetary disk before the latter disperses (e.g. Goldreich \& Tremaine 1980). Migration could also occur by the Kozai-Lidov mechanism increasing the eccentricity of the planet followed by tidal circularization (Kozai 1962; Lidov 1962; Wu \& Murrav 2003; Takeda \& Rasio 2005; Nagasawa, Ida \& Bessho 2008; Perets \& Fabrycky 2009). Evidence obtained by the RossiterMcLaughlin effect suggests that some fraction of the hot Jupiters may have been produced through dynamical interactions (see e.g. Winn et al. 2005; Lubow \& Ida 2010; Triaud et al. 2010; (Albrecht et al. 2012). Hot-Jupiter planets dominated the initial planet discoveries because they are large and close to their host star. However, we now know that they are quite rare and orbit only about one percent of solar type stars (e.g. Wright et al. 2012).

Opinions vary on whether in the Solar System Jupiter has significantly migrated. On one hand Morbidelli et al. (2010) 

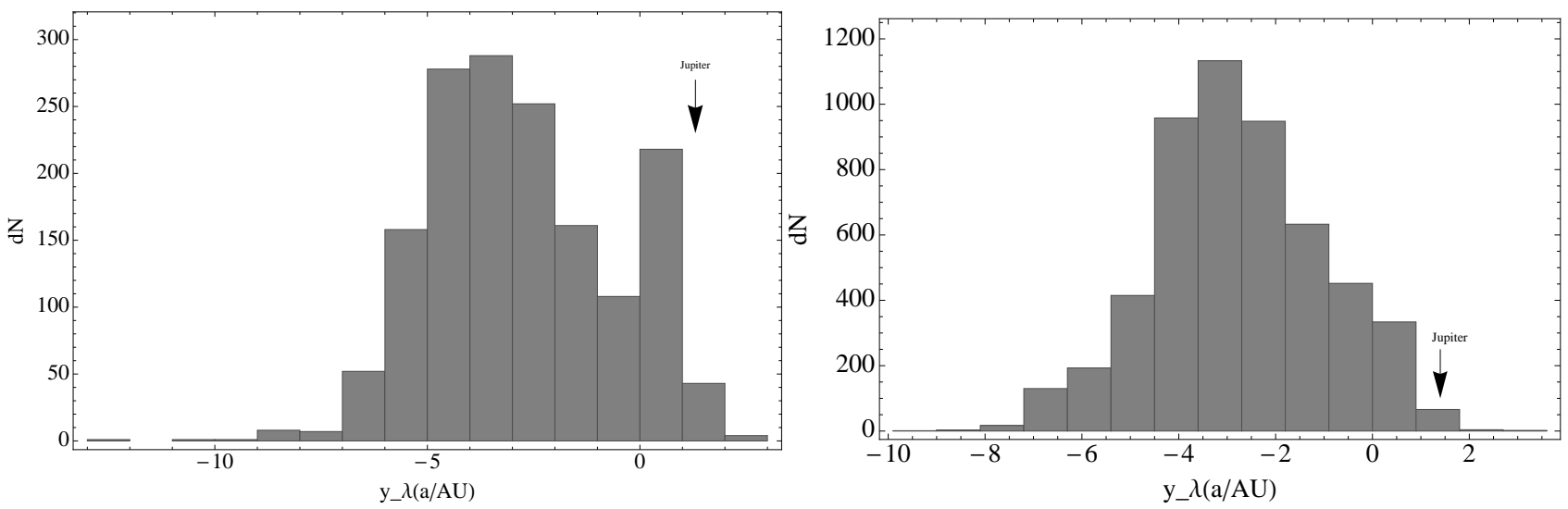

FIG. 2.- Box-Cox transformed distribution of exoplanet semi-major axis. Left: Only including confirmed exoplanets. The total number of exoplanets is 1580 . Right: Including all planet candidates. The total number of exoplanets is 5289.

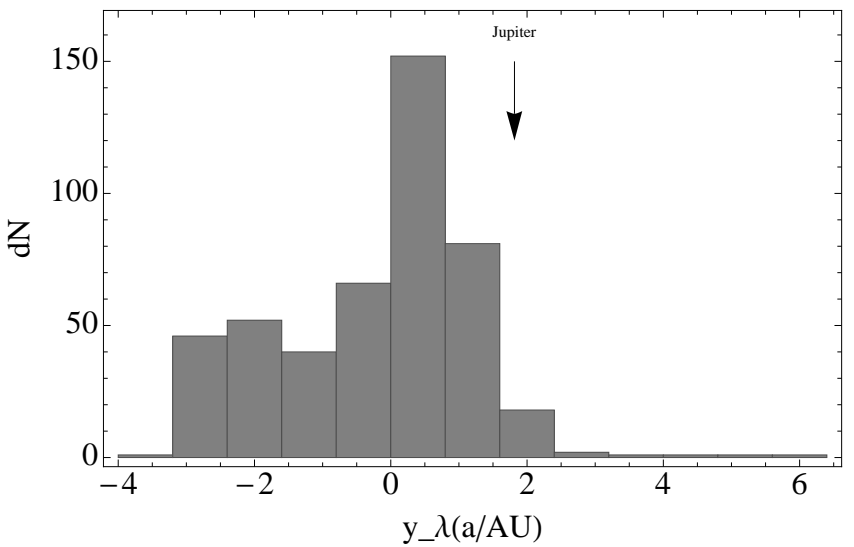

FIG. 3.- Box-Cox transformed distribution of exoplanet semi-major axis not including planets found by the transit method. The total number of exoplanets is 473 .

suggested that Jupiter did not migrate much from its formation location, and on the other, Walsh et al. (2011) proposed that the low mass of Mars could be explained by gas-driven early migration of Jupiter. Batygin \& Laughlin (2015) further suggested that this formation process could explain the lack of objects in our inner Solar System. Armitage et al. (2002) assumed that planets form constantly at a radius of $5 \mathrm{AU}$ and found theoretically that about 10 to $15 \%$ of systems will have a Jupiter mass planet that does not migrate significantly. However, this conclusion will be affected by the presence of a dead zone (a region of the disk with no turbulence, e.g. Gammie 1996; Martin et al. 2012a, b) that may slow or halt migration altogether. Furthermore, the inner and outer edges of a dead zone may act as planet traps that stop migration (e.g. Hasegawa \& Pudritz 2011, 2013). Thus, the distribution of planet semi-major axes definitely does not represent the initial distribution at the time of planet formation.

Given that Jupiter mass planets are thought to form in the vicinity of Jupiter's current radial location, theory suggests that the radial location of Jupiter is not particularly special. The current observational bias towards planets that are close to their host star means that it is easier to find planets that have migrated inwards, rather than those that have not, or even those that may have migrated outwards. We expect in the future that with more complete observations of Jupiter mass planets at Jupiter's radial location we will be able to constrain the migration mechanisms and uncover how special Jupiter really is for its small (net) distance of migration.

\section{PLANETARY PROPERTIES}

In this Section we consider how the masses and densities of the planets in our Solar System compare to those in exosolar systems. In this context, we discuss also the potential significance of the lack of a super-Earth in our Solar System.

\subsection{Planet Masses}

Fig. 4 shows the distribution of the approximate masses of the exoplanets that have been observed to dat $\square$. The masses of the planets within our Solar System are shown with arrows at the top (but are not included in the data). The masses of the gas giants fit well with those of exosolar planets, but the terrestrial planets are all on the low side. This is most likely due (at least partially) to the difficulty in finding low-mass planets. The masses of the exoplanets are strongly biased towards high-mass and short-period planets. Kepler has shown us that small planets are very common but the mass measurement of small mass planets is difficult and thus currently they appear to be rare.

There are two peaks in the data, the first of which is at a mass between that of the Earth and that of Uranus, at around $0.01 M_{\mathrm{J}}$, where $M_{\mathrm{J}}$ is the mass of Jupiter. Planets with a mass in the range of $1 M_{\oplus}$ to $10 M_{\oplus}$ (where $M_{\oplus}$ is the mass of the Earth) are known as super-Earths (e.g. Valencia, Sasselov \& O'Connell 2007). Our Solar System does not contain any super-Earths thus in that sense it is somewhat unusual. We discuss this further in subsection 3.3. The second peak is around the mass of Jupiter.

We fit the exoplanet mass data with a binormal probability density function (PDF)

$$
P(z) d z \propto \frac{1}{\sigma_{1}} \mathrm{e}^{\frac{\left(z-\mu_{1}\right)^{2}}{\sigma_{1}^{2}}}+\frac{w_{2}}{\sigma_{2}} \mathrm{e}^{\frac{\left(z-\mu_{2}\right)^{2}}{\sigma_{2}^{2}}},
$$

where $z=\log _{10}(m)$. With a Kolmogorov-Smirnov (KS) test we find the best fitting parameters to be $\mu_{1}=-1.80, \sigma_{1}=$ $0.28, w_{2}=0.69, \mu_{2}=0.12, \sigma_{2}=0.56$ and we show the PDF as the solid line in Fig. 4. With this distribution, we find that Jupiter is very typical at only $-0.26 \sigma$ from the higher-mass peak, while Saturn is at $-1.3 \sigma$. The terrestrial planets are hard to compare because there are so few data points for those

1 The masses for planets that have been observed by the Doppler method are $M_{\mathrm{p}} \sin i$, where $i$ is the orbital inclination. For directly imaged planets, the mass is predicted by theoretical models of the planets' evolution. For the planets that have been observed by microlensing, it's the ratio of the planet to star mass that is measured with accuracy. 


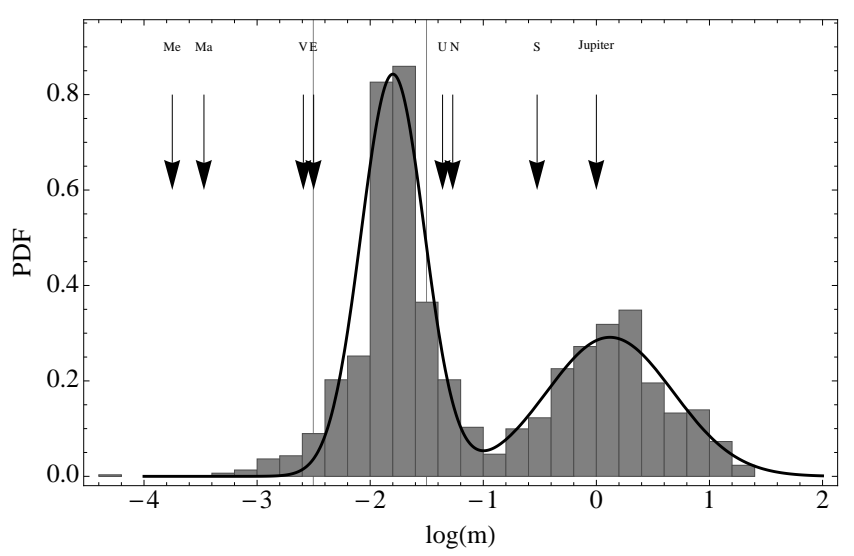

FIG. 4.- Exoplanet mass distribution. The arrows show the masses of the planets in our Solar System. The vertical lines shows range of planets that are considered to be super-Earths, the lower limit is the mass of the Earth and the upper limit is the lower limit to the mass of a giant planet at $10 M_{\oplus}$. The total number of exoplanets is 1516 .

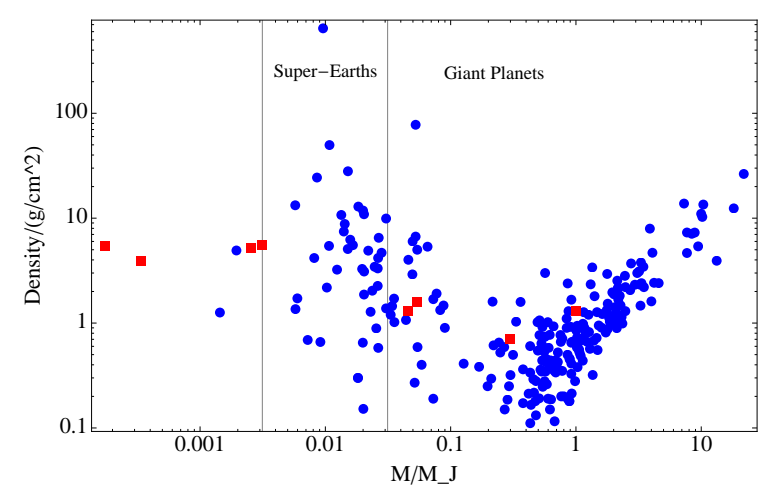

FIG. 5.- Densities of planets as a function of their mass. The blue circles show the exoplanets (total number of 287) and the red squares the planets in our Solar System. The vertical lines shows range of planets that are considered to be super-Earths, the lower limit is the mass of the Earth and the upper limit is the lower limit to the mass of a giant planet at $10 \mathrm{M}_{\oplus}$.

small masses. However, Neptune lies at $1.89 \sigma$ and Uranus at $1.57 \sigma$ from the lower-mass peak.

Gas giants are thought to form outside of the snow line radius in the protoplanetary disk where there is more solid material available to form massive planets (e.g. Pollack et al. 1996; Martin \& Livio 2012, 2013a). The surface density of the protoplanetary disk decreases with increasing distance from the star and the timescale to form a planet increases with radius. Thus, lower mass gas giants could preferentially form farther away from the star. Low mass and large orbital radius planets are certainly harder to detect than those with higher mass and lower orbital radius. This can explain the lack of observed small mass planets, but also perhaps the dip in the observed distribution. For example, if Uranus and Neptune were around another star, at their large orbital radii, they would also be difficult to detect. The only method that could currently detect them is direct imaging. However, the smallest mass planet that has been found with this method is Formaulhaut $b$ that has an approximate mass of $\lesssim 2 \mathrm{M}_{\mathrm{J}}$ (Currie et al. 2012). It therefore remains a possibility that the double peaked mass distribution is solely the result of selection effects.

\subsection{Planet Densities}

Fig. 5 shows the approximate densities of the observed planets as a function of their mass. The exoplanets are shown in blue and the planets in our Solar System in red. While the lower mass exoplanets have a large range in their density for a given mass (see also Wolfgang \& Laughlin 2012; Marcy et al. 2014a; Howe, Burrows \& Verne 2014; Knutson et al. 2014), the giant planets show a clear correlation of increasing density with mass. Thus, the super-Earths may have a wide range of compositions Valencia, Sasselov \& O'Connell 2007). Despite the large spread in the data for the low mass planets, there appears to be a trend of decreasing density with increasing mass. This could be attributed qualitatively to the peak in the theoretical radius against mass of a planet (see e.g. Zapolsky \& Salpeter 1969; Seager et al. 2007; Fortney, Marley \& Barnes 2007; Mordasini et al. 2012).

More recently, it has been suggested that the super-Earths (with radii in the range $1 \mathrm{R}_{\oplus}<R<4 \mathrm{R}_{\oplus}$ ) show two trends separated by a critical planet radius. The smallest planets increase in density with radius while those that are larger decrease suggesting that the larger planets have a large amount of volatiles on a rocky core. There is some uncertainty over the value of the critical radius, as estimates range from about $1.5 R_{\oplus}$ to $2 \mathrm{R}_{\oplus}$ Petigura, Marcy \& Howard (2013); Lopez \& Fortney (2014); Weiss \& Marcy (2014); Marcy et al. (2014b), if it exists at all (Morton \& Swift 2014).

The data suggest that the densities of the giant planets within our Solar System are very typical of those of observed exoplanets. The masses of the terrestrial planets in our Solar System are on the edge of our current sensitivity and thus it is hard to draw any conclusions about their densities. However, recently, Dressing et al. (2015) found that the Earth (and Venus) can be modelled with the same ratio of iron to magnesium silicate as the low mass exoplanets observed and thus the Earth may not be special in this respect.

\subsection{Lack of a super-Earth}

It is interesting to examine whether our Solar System's lack of a super-Earth is truly unusual. There have been several attempts to calculate an occurrence rate for super-Earths taking into account the selection biases. The results for RV observations predict an occurrence rate in the range $10-20 \%$ in the period range $P_{\mathrm{b}}<50 \mathrm{~d}$ (Howard et al. 2010; Mayor et al. 2011). The transiting planet observations imply a range of occurrence rates that is at most as high as $50 \%$ in the period range $P_{\mathrm{b}}<85 \mathrm{~d}$ (Fressin et al. 2013). More recently Burke et al. (2015) examined the Kepler sample for planets with radii in the range $0.75<R<2.5 R_{\oplus}$ with orbital periods in the range 50 to $300 \mathrm{~d}$ and found an occurrence rate of $77 \%$. Although these results include Earth-size planets and some of the periods are longer than that of Mercury, the occurrence rate increases with short orbital periods, making the existence of close-in planets more likely. The high occurrence rate of these types of planets offers perhaps the strongest argument against the Solar System being very common, but even that does not necessarily make it extremely rare. Typically, systems that have an observed super-Earth, have more than one and this is theoretically expected if the planets form by mergers of inwardly migrating cores (e.g. Terquem \& Papaloizou 2007; Cossou et al. 2014).

It is possible that the presence of a super-Earth can affect terrestrial planet formation. Many of the super-Earths observed are at small radial locations, where theoretically they could not have formed (e.g. Raymond \& Cossou 2014; Schlichting 2014). Izidoro, Morbidelli \& Raymond (2014) found that if a super-Earth migrates sufficiently slowly through the habitable zone (defined as the radial range of dis- 


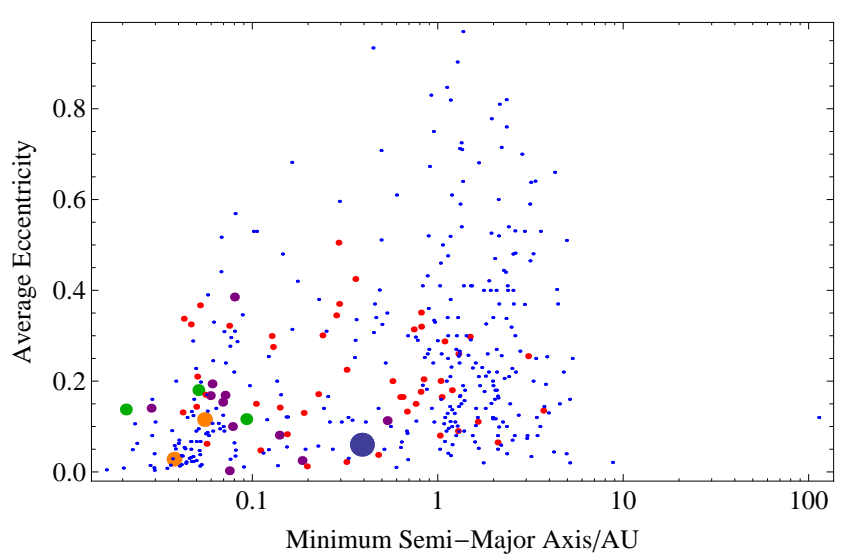

FIG. 6.- The average eccentricity in a planetary system vs the smallest planet semi-major axis. The size of the point is proportional to the number of planets in the system. The small blue points have 1 planet, the red points have 2 planets, the purple have 3 , the green have 4, the orange have 5 and the Solar System with 8 is shown in the large blue point. The Solar System has an average eccentricity of 0.06 and Mercury, being the inner most planet, at $0.39 A U$.

tances from the star at which a rocky planet can maintain liquid water on its surface) then any terrestrial planet that later forms there would be volatile-rich and not very Earth-like.

In conclusion, the masses and densities of the planets of our Solar System appear to be very typical of those of exoplanets. However, the lack of a planet with a mass in the range $1-10 M_{\oplus}$, a super-Earth, makes the Solar System appear somewhat special.

\section{DISCUSSION}

Generally, the physical properties of the planets in our Solar System are quite typical when compared to those of the observed exoplanets, although the lack of a super-Earth is unusual. The orbital properties, however, may be somewhat special and perhaps more conducive to life. Low eccentricity planets have a more stable temperature throughout the orbit and therefore may be more likely to host life (e.g. Williams \& Pollard 2002; Gaidos \& Williams 2004). Furthermore, planetary systems with a low average eccentricity are more likely to have long term dynamical stability. For example, the terrestrial planets in our Solar System are expected to be dynamically stable at least until the Sun becomes a red giant and engulfs the inner planets (e.g. Laughlin 2009).

There are a few other factors that could, in principle at least, make our Solar System special with respect to the emergence of life. First we can consider the age. The current age of our Sun is about half the age of the disk of our Galaxy, and also half of the Sun's total lifetime. Thus, we expect that roughly half of the stars in our Galaxy's disk are older and half are younger than our Sun. This implies that the age of our Solar System is definitely not special. Furthermore, Behroozi \& Peeples (2015) considered the planet formation history of the Milky way and determined that our Solar System formed close to the median epoch for giant planet formation. They also found that about $80 \%$ of the currentlyexisting Earth-like planets were already formed at the time of the Earth's formation. We also note that the fact that the Solar System contains a single star does not make it particularly special, since the binary fraction in the Kepler sample, for example, is about $50 \%$ (Horch et al. 2014).

The presence of terrestrial planets in the habitable zone around their host star appears to be quite common. For example, Dressing \& Charbonneau (2015) examined the Kepler data for $\mathrm{M}$ dwarfs and found that for orbital periods shorter than $50 \mathrm{~d}$, the occurrence rate of Earth-size planets in the habitable zone is around $18-27 \%$. This conservative estimate could be as high as $\sim 50 \%$ depending on how the habitable zone is defined. This is consistent with radial velocity surveys that find 0.41 potentially habitable planets per $\mathrm{M}$ dwarf (Bonfils et al. 2013). Thus, an Earth-size planet in a habitable zone is not uncommon.

A habitable planet may require a large moon which in turn may require an asteroid collision (Canup \& Asphaug 2001). Thus, systems which contain an asteroid belt may be more conducive to initiating life. However, habitability may be sensitive to the size of the asteroid belt (Martin \& Livio 2013b). As we have noted in the Introduction, asteroid belts could be a common feature of planetary systems (Chen et al. 2014).

The metallicity of a protoplanetary disk (and hence the host star) determines the structure of a planetary system that forms (e.g. Buchhave et al. 2014; Wang \& Fischer 2015). The higher the metallicity of a star, the more giant planets that are observed (e.g. Fischer \& Valenti 2005; Sousa et al. 2011; Gonzalez 2014; Reffert et al. 2015). However, the correlation for lower mass planets is unclear (Buchhave et al. 2012; Mayor, Lovis \& Santos 2014). Planets with radii less than four times that of the Earth are observed around stars with a wide range of metallicities. However, the average metallicity of stars hosting small planets $\left(R_{\mathrm{p}}<1.7 R_{\oplus}\right)$ in Buchhave et al. (2014) is very close to solar. Although such planets can form at a wide range of metallicities, the fact that the average metallicity of the small planets is solar may not be a coincidence. Thus, while the metallicity of our Solar System may not be especially promotive to the formation of a habitable planet, it's unclear whether the Solar System is special or not in this respect.

The variability of our Sun has been compared to the activity of stars in the Kepler sample with conflicting conclusions. Basri et al. (2010) and Basri, Walkowicz \& Reiners (2013) found that the Sun is rather typical with only a quarter to a third of stars in the Kepler sample being more active than the Sun. On the other hand, McQuillan, Aigrain \& Roberts (2012) found the Sun to be relatively quiet with $60 \%$ of stars being more active. The difference in the conclusions stems from choices in defining the activity level of our Sun and the inclusion of stars with fainter magnitudes in McQuillan, Aigrain \& Roberts (2012). However, the studies agree that the active fraction of stars becomes larger for cooler stars. M dwarfs have a fraction of $90 \%$ that are more active than the Sun. Thus, compared to other Sun-like stars, our Sun could be typical, but compared to cooler stars, our Sun is certainly quiet.

In general, there are three aspects in which the Solar System differs most from other observed multi-planet systems. First, the low mean eccentricity of the planets in the Solar System maybe somewhat special, although this may be accounted for by selection effects. Secondly, there is in the total lack of planets inside Mercury's orbit. Massive planets migrating through the habitable zone can change the course of planet formation in that region. Overall, however, processes that could act to clear the inner part of the Solar Systems (such as giant planet migration), are believed to be operating within a non-negligible fraction of the exoplanet systems (e.g. Batygin \& Laughlin 2015). Third, the lack of super-Earths in our Solar System is somewhat special and could have allowed 
the Earth to become habitable. A close-in super-Earth could also affect the dynamical stability of a terrestrial planet in the habitable zone and this should be investigated in future work.

We consider the first two of these special parameters in more detail in Fig. 6 For planets with measured eccentricity, we plot the mean eccentricity of the planetary system and the semi-major axis of the innermost planet observed within the system. In this parameter space, the Solar System appears to be somewhat special, but far from being rare. Although, most of the systems with three or more planets, do have a planet with an orbital semi-major axis smaller than that of Mercury, this could be at least partly due to selection effects. The observed semi-major axis of the innermost planet may be close to complete but the number of planets in each system is certainly not. If the innermost planet is very close in, then it is easier to detect the planets outside of its orbit. If on the other hand the innermost planet is farther out (e.g. some of the small blue and red points in Fig. 6) then additional planets will be difficult to find (see also discussion in Section 2.2).

There are many factors that may be required in order to form a habitable planet. When we multiply the probabilities for each together, we may end up with a small probability for such an event. However, since we currently do not know which factors are truly important for life to emerge, such an exercise does not make much sense. If we consider too many details, clearly the Solar System is special because all systems are different. However, at the moment we have not identified any parameter that makes the Solar System so significantly different that it would make it rare.

\section{CONCLUSIONS}

We find that the properties of the planets in our Solar System are not so significantly special compared to those in ex- osolar systems to make the Solar System extremely rare. The masses and densities are typical, although the lack of a superEarth sized planet appears to be somewhat unusual. The orbital locations of our planets seem to be somewhat special but this is most likely due to selection effects and the difficulty in finding planets with a small mass or large orbital period. The mean semi-major axis of observed exoplanets is smaller than the distance of Mercury to the Sun. The relative depletion in mass of the Solar System's terrestrial region may be important. The eccentricities are relatively low compared to observed exoplanets, although the observations are biased towards finding high eccentricity planets. The low eccentricity, however, may be expected for multi-planet systems. Thus, the two characteristics of the Solar System that we find to be most special are the lack of super-Earths with orbital periods of days to months and the general lack of planets inside of the orbital radius of Mercury.

From the perspective of habitability the Solar System does not appear to be particularly special. If exosolar life happens to be rare it would probably not be because of simple basic physical parameters, but because of more subtle processes that are related to the emergence and evolution of life. Since at the moment we don't know what those might be, we can allow ourselves to be optimistic about the prospects of detecting exosolar life. We should make every possible effort to detect and characterise the atmospheres of a few dozen Earth-size planets in the habitable zone, in the coming two decades.

\section{ACKNOWLEDGEMENTS}

We thank the anonymous referee for useful comments. This research has made use of the Exoplanet Orbit Database and the Exoplanet Data Explorer at exoplanets.org.

\section{REFERENCES}

Albrecht S. et al., 2012, ApJ, 757, 18

Armitage P. J., Livio M., Lubow S. H., Pringle J. E., 2002, MNRAS, 334, 248

Basri G. et al., 2010, ApJl, 713, L155

Basri G., Walkowicz L. M., Reiners A., 2013, ApJ, 769, 37

Batalha N. M., 2014, PNAS, 111, 12647

Batygin K., Laughlin G., 2015, Proceedings of the National Academy of Science, 112, 4214

Beer M. E., King A. R., Livio M., Pringle J. E., 2004, MNRAS, 354, 763

Behroozi P. S., Peeples M. S., 2015, MNRAS, submitted

Bodenheimer P., Hubickyj O., Lissauer J. J., 2000, Icarus, 143, 2

Bonfils X. et al., 2013, A\&A, 549, A109

Box G. E. P., Cox D. R., 1964, J. Roy. Stat. Soc., Ser. B, 26, 211

Buchhave L. A. et al., 2014, Nature, 509, 593

Buchhave L. A. et al., 2012, Nature, 486, 375

Burke C. J. et al., 2015, ArXiv e-prints

Cabrera J. et al., 2014, ApJ, 781, 18

Canup R. M., Asphaug E., 2001, Nat, 412, 708

Cassan A., Kubas D., Beaulieu J.-P., et al., 2012, Nat, 481, 167

Chen C. H., Mittal T., Kuchner M., Forrest W. J., Lisse C. M., Manoj P., Sargent B. A., Watson D. M., 2014, ApJS, 211, 25

Cossou C., Raymond S. N., Hersant F., Pierens A., 2014, A\&A, 569, A56

Currie T. et al., 2012, ApJL, 760, L32

Désert J.-M. et al., 2015, ApJ, 804, 59

Dressing C. D., Charbonneau D., 2015, ArXiv e-prints

Dressing C. D. et al., 2015, ApJ, 800, 135

Fischer D. A., Valenti J., 2005, ApJ, 622, 1102

Fortney J. J., Marley M. S., Barnes J. W., 2007, ApJ, 659, 1661

Fressin F. et al., 2013, ApJ, 766, 81

Gaidos E., Williams D. M., 2004, New Astronomy, 10, 67

Gammie C. F., 1996, ApJ, 457, 355

Goldreich P., Tremaine S., 1980, ApJ, 241, 425

Gonzalez G., 2014, MNRAS, 443, 393

Gould A., Dong S., Gaudi, et al., 2010, ApJ, 720, 1073

Han E., Wang S. X., Wright J. T., Feng Y. K., Zhao M., Fakhouri O., Brown

J. I., Hancock C., 2014, PASP, 126, 827

Hasegawa Y., Pudritz R. E., 2011, MNRAS, 417, 1236

Hasegawa Y., Pudritz R. E., 2013, ApJ, 778, 78
Hogg D. W., Myers A. D., Bovy J., 2010, ApJ, 725, 2166

Horch E. P., Howell S. B., Everett M. E., Ciardi D. R., 2014, ArXiv e-prints Howard A. W. et al., 2010, Science, 330, 653

Howe A. R., Burrows A., Verne W., 2014, ApJ, 787, 173

Izidoro A., Morbidelli A., Raymond S. N., 2014, ApJ, 794, 11

Kane S. R., Ciardi D. R., Gelino D. M., von Braun K., 2012, MNRAS, 425, 757

Kennedy G. M., Wyatt M. C., 2014, MNRAS, 444, 3164

Knutson H. A. et al., 2014, ApJ, 794, 155

Kozai Y., 1962, AJ, 67, 591

Lafrenière D., Jayawardhana R., van Kerkwijk M. H., 2010, ApJ, 719, 497

Laughlin G., 2009, Nature, 459, 781

Lidov M. L., 1962, Planet. Space Sci., 9, 719

Limbach M. A., Turner E. L., 2014, ArXiv e-prints

Lissauer J. J., Dawson R. I., Tremaine S., 2014, Nature, 513, 336

Lopez E. D., Fortney J. J., 2014, ApJ, 792, 1

Lubow S. H., Ida S., 2010, Planet Migration, Seager S., ed., pp. 347-371

Marcy G. W. et al., 2014a, ApJS, 210, 20

Marcy G. W., Weiss L. M., Petigura E. A., Isaacson H., Howard A. W.,

Buchhave L. A., 2014b, Proceedings of the National Academy of Science, 111,12655

Marois C., Macintosh B., Barman T., Zuckerman B., Song I., Patience J., Lafrenière D., Doyon R., 2008, Science, 322, 1348

Martin R. G., Livio M., 2012, MNRAS, 425, L6

Martin R. G., Livio M., 2013a, MNRAS, 434, 633

Martin R. G., Livio M., 2013b, MNRAS, 428, L11

Martin R. G., Lubow S. H., Livio M., Pringle J. E., 2012a, MNRAS, 420, 3139

Martin R. G., Lubow S. H., Livio M., Pringle J. E., 2012b, MNRAS, 423, 2718

Mayor M., Lovis C., Santos N. C., 2014, Nature, 513, 328

Mayor M. et al., 2011, ArXiv e-prints

McQuillan A., Aigrain S., Roberts S., 2012, A\&A, 539, A137

Moorhead A. V. et al., 2011, ApJS, 197, 1

Morbidelli A., Brasser R., Gomes R., Levison H. F., Tsiganis K., 2010, AJ, 140,1391

Mordasini C., Alibert Y., Georgy C., Dittkrist K.-M., Klahr H., Henning T., 2012, A\&A, 547, A112 
Morton T. D., Swift J., 2014, ApJ, 791, 10

Nagasawa M., Ida S., Bessho T., 2008, ApJ, 678, 498

Panić O. et al., 2013, MNRAS, 435, 1037

Perets H. B., Fabrycky D. C., 2009, ApJ, 697, 1048

Petigura E. A., Marcy G. W., Howard A. W., 2013, ApJ, 770, 69

Pollack J. B., Hubickyj O., Bodenheimer P., Lissauer J. J., Podolak M., Greenzweig Y., 1996, Icarus, 124, 62

Raymond S. N., Cossou C., 2014, MNRAS, 440, L11

Reffert S., Bergmann C., Quirrenbach A., Trifonov T., Künstler A., 2015, A\&A, 574, A116

Rowe J. F., Bryson S. T., Marcy G. W., et al., 2014, ApJ, 784, 45

Schlichting H. E., 2014, ApJL, 795, L15

Seager S., Kuchner M., Hier-Majumder C. A., Militzer B., 2007, ApJ, 669, 1279

Shen Y., Turner E. L., 2008, ApJ, 685, 553

Sousa S. G., Santos N. C., Israelian G., Mayor M., Udry S., 2011, A\&A, 533, A141

Takeda G., Rasio F. A., 2005, ApJ, 627, 1001

Terquem C., Papaloizou J. C. B., 2007, ApJ, 654, 1110

Triaud A. H. M. J. et al., 2010, A\&A, 524, A25
Valencia D., Sasselov D. D., O’Connell R. J., 2007, ApJ, 665, 1413

Van Eylen V., Albrecht S., 2015, ArXiv e-prints

Velilla S., 1993, Statiscics and Probability Letters, 17, 259

Walsh K. J., Morbidelli A., Raymond S. N., O'Brien D. P., Mandell A. M., 2011, Nature, 475, 206

Wang J., Fischer D. A., 2015, AJ, 149, 14

Weiss L. M., Marcy G. W., 2014, ApJl, 783, L6

Williams D. M., Pollard D., 2002, International Journal of Astrobiology, 1, 61

Winn J. N., Fabrycky D. C., 2014, ArXiv e-prints

Winn J. N. et al., 2005, ApJ, 631, 1215

Wolfgang A., Laughlin G., 2012, ApJ, 750, 148

Wright J. T. et al., 2011, PASP, 123, 412

Wright J. T., Marcy G. W., Howard A. W., Johnson J. A., Morton T. D., Fischer D. A., 2012, ApJ, 753, 160

Wu Y., Murray N., 2003, ApJ, 589, 605

Wyatt M. C., 2008, ARA\&A, 46, 339

Zakamska N. L., Pan M., Ford E. B., 2011, MNRAS, 410, 1895

Zapolsky H. S., Salpeter E. E., 1969, ApJ, 158, 809 